\title{
Groups with Finitely Many Normalizers of Non-polycyclic Subgroups
}

\author{
Maria De Falco Francesco de Giovanni ${ }^{\dagger}$ Carmela Musella \\ Dipartimento di Matematica e Applicazioni, Università di Napoli Federico II \\ Complesso Universitario Monte S. Angelo, Via Cintia, I-80126 Napoli, Italy \\ E-mail: mdefalco@unina.it degiovan@unina.it cmusella@unina.it
}

Received 15 March 2007

Communicated by A.C. Kim

\begin{abstract}
The structure of locally graded groups with finitely many normalizers of nonpolycyclic subgroups is investigated. In particular, it is proved that such groups either are polycyclic or have Černikov commutator subgroups.
\end{abstract}

2000 Mathematics Subject Classification: 20F24

Keywords: polycyclic subgroup, normalizer

\section{Introduction}

In a famous paper of 1955, Neumann [12] proved that each subgroup of a group $G$ has finitely many conjugates if and only if the centre $Z(G)$ has finite index, and hence, central-by-finite groups are precisely those groups in which the normalizers of subgroups have finite index. This result suggests that the behaviour of normalizers has a strong influence on the structure of the group. In fact, it follows easily from a result of Polovickiı [13] that a group has finitely many normalizers of abelian subgroups if and only if it is central-by-finite. In a series of relevant papers, Romalis and Sesekin (see [15], [16], [17]) investigated (generalized) soluble groups in which all non-normal subgroups are abelian, and proved in particular that such groups have finite commutator subgroups. This result has been recently extended to the case of groups with finitely many normalizers of non-abelian subgroups (see [3]). Moreover, groups with finitely many normalizers of subgroups with a given property $\chi$ have been studied for several other different choices of $\chi$ (see [4], [5], [6]).

The aim of this paper is to study groups with finitely many normalizers of non-polycyclic subgroups. The structure of groups in which every non-normal subgroup is polycyclic has been completely described in [8], while the case of groups for which the set of all subgroups which are neither normal nor polycyclic is finite has been considered in [2]. We will work within the universe of locally graded

${ }^{\dagger}$ Corresponding author. 
groups, i.e., groups in which every finitely generated non-trivial subgroup contains a proper subgroup of finite index. This is a quite large class, containing in particular all locally soluble-by-finite groups. Our main result shows that any locally graded group with finitely many normalizers of non-polycyclic subgroups either is polycyclic or has a Černikov commutator subgroup. In particular, residually finite non-polycyclic groups with the above property have finite commutator subgroups. Moreover, torsion-free locally graded groups with finitely many normalizers of nonpolycyclic subgroups are either polycyclic or abelian.

Most of our notation is standard and can be found in [14].

\section{Statements and Proofs}

Our first result shows that any group with finitely many normalizers of non-polycyclic subgroups contains a subgroup of finite index in which every non-polycyclic subgroup is subnormal with defect at most 2 .

Lemma 2.1. Let $G$ be a group with finitely many normalizers of non-polycyclic subgroups. Then $G$ contains a characteristic subgroup $M$ of finite index such that $N_{M}(X)$ is normal in $M$ for each non-polycyclic subgroup $X$ of $M$.

Proof. If $X$ is any non-polycyclic subgroup of $G$, its normalizer $N_{G}(X)$ has obviously finitely many images under automorphisms of $G$. In particular, the subgroup $N_{G}(X)$ has finitely many conjugates in $G$ and so the index $\left|G: N_{G}\left(N_{G}(X)\right)\right|$ is finite. It follows that the characteristic subgroup

$$
M(X)=\bigcap_{\alpha \in \operatorname{Aut}(G)} N_{G}\left(N_{G}(X)\right)^{\alpha}
$$

also has finite index in $G$. Let $\mathcal{H}$ be the set of all non-polycyclic subgroups of $G$. If $X$ and $Y$ are elements of $\mathcal{H}$ such that $N_{G}(X)=N_{G}(Y)$, then $M(X)=M(Y)$, and hence

$$
M=\bigcap_{X \in \mathcal{H}} M(X)
$$

is a characteristic subgroup of finite index of $G$. Let $X$ be any non-polycyclic subgroup of $M$. Then $M \leq M(X) \leq N_{G}\left(N_{G}(X)\right)$, and so the normalizer $N_{M}(X)=$ $N_{G}(X) \cap M$ is a normal subgroup of $M$.

Lemma 2.2. Let $G$ be a locally graded group with finitely many normalizers of non-polycyclic subgroups. Then $G$ is soluble-by-finite and locally satisfies the maximal condition on subgroups.

Proof. By Lemma 2.1, the group $G$ contains a characteristic subgroup $M$ of finite index in which all normalizers of non-polycyclic subgroups are normal. Let $X$ be any non-polycyclic subgroup of $M$. Then every subgroup of $M$ containing $X$ is subnormal with defect at most 2 , so that both groups $N_{M}(X) / X$ and $M / N_{M}(X)$ are nilpotent with class at most 3 (see [10, Theorem 1]). In particular, $M^{(6)}$ is contained in $X$, and hence, every proper subgroup of $M^{(6)}$ is polycyclic. Thus, $M^{(6)}$ is either soluble or finite (see [8, Corollary 2.6]), so $G$ is soluble-by-finite. 
In order to prove that $G$ locally satisfies the maximal condition on subgroups, we may obviously suppose that $G$ is finitely generated, so that $M$ is also finitely generated. If every subgroup of finite index of $M$ is subnormal, the group $M$ is nilpotent (see [14, Part 2, Theorem 10.51]) and $G$ satisfies the maximal condition. Now assume that $M$ contains a non-subnormal subgroup $H$ of finite index. It follows from the definition of $M$ that $H$ is polycyclic, so that in this case $G$ also satisfies the maximal condition on subgroups.

It follows in particular from Polovickiü's theorem quoted in the introduction that any group with finitely many normalizers has a finite commutator subgroup. Our next lemma is an obvious corollary of this fact.

Lemma 2.3. Let $G$ be a group with finitely many normalizers of non-polycyclic subgroups, and let $N$ be a normal subgroup of $G$ which is not polycyclic. Then $G^{\prime} /\left(G^{\prime} \cap N\right)$ is finite.

For our purposes, we also need a result of Neumann [11], that actually holds in the more general situation of groups covered by cosets of subgroups.

Lemma 2.4. Let the group $G=X_{1} \cup \cdots \cup X_{t}$ be the union of finitely many subgroups $X_{1}, \ldots, X_{t}$. Then any $X_{i}$ of infinite index can be omitted from this decomposition, in particular, at least one of the subgroups $X_{1}, \ldots, X_{t}$ has finite index in $G$.

Recall that a group $G$ is said to be an $F C$-group if each element of $G$ has finitely many conjugates, or equivalently, if the centralizer $C_{G}(g)$ has finite index in $G$ for every $g \in G$. In particular, any group with finite commutator subgroup has the property $F C$. Moreover, it is also clear that abelian-by-finite $F C$-groups are central-by-finite.

Lemma 2.5. Let $G$ be an $F C$-group with finitely many normalizers of non-polycyclic subgroups. Then the factor group $G / Z(G)$ is finite.

Proof. Assume for a contradiction that $G / Z(G)$ is infinite, so that $G$ contains a subgroup which is not finitely generated and has infinitely many conjugates (see [12]). Let $N_{G}\left(X_{1}\right), \ldots, N_{G}\left(X_{k}\right)$ be the normalizers of infinite index of non-polycyclic subgroups of $G$, then $N_{G}\left(X_{1}\right) \cup \cdots \cup N_{G}\left(X_{k}\right)$ is a proper subset of $G$ by Neumann's Lemma, and we may consider an element

$$
g \in G \backslash \bigcup_{i=1}^{k} N_{G}\left(X_{i}\right) .
$$

Then each subgroup of $C_{G}(g)$ has finitely many conjugates, and hence, $C_{G}(g)$ is a central-by-finite group (see [12]). As the index $\left|G: C_{G}(g)\right|$ is finite, it follows that $G$ is abelian-by-finite and so even central-by-finite. This contradiction proves the lemma.

Lemma 2.6. Let $G$ be a torsion-free soluble group with a unique proper normalizer of non-polycyclic subgroups. Then $G$ is nilpotent. 
Proof. Assume for a contradiction that the group $G$ is not nilpotent. Clearly, all non-polycyclic subgroups of $G$ are subnormal and so there exists an element $g$ of $G$ such that $\langle g\rangle$ is not subnormal in $G$ (see [1] or [18]). Let $X$ be a non-polycyclic subgroup of $G$ whose normalizer $N_{G}(X)$ is properly contained in $G$. Since all subgroups of $N_{G}(X)$ are either polycyclic or normal, we have that $N_{G}(X)$ is abelian (see [8]). Then $N_{G}(X)$ is finitely generated as a $\langle g\rangle$-module (see [9, Lemma 15]), and hence, the subgroup $\left\langle a, N_{G}(X)\right\rangle$ is finitely generated. Thus, $\left\langle a, N_{G}(X)\right\rangle$ is polycyclic by Lemma 2.2 , and this contradiction proves the lemma.

Our next result is an easy consequence of Dietzmann's Lemma.

Lemma 2.7. Let $G$ be a group and let $X$ be a periodic subgroup of $G$. If $X$ has finitely many conjugates in $G$, then the normal closure $X^{G}$ is periodic.

Proof. Since $X$ has finitely many conjugates, there exists a normal subgroup $K$ of $G$ such that the index $|G: K|$ is finite and $X^{K}=X$. Then $X \cap K$ is contained in the largest periodic normal subgroup $L$ of $K$. Moreover, $X L / L$ is a finite subgroup of $G / L$ with finitely many conjugates and hence, it follows from Dietzmann's Lemma that $X^{G} L / L$ is finite. Therefore, the subgroup $X^{G}$ is periodic.

The following lemma on groups whose subgroups are either subnormal or finitely generated has been proved in [9, p. 217]. Recall that the Baer radical of a group $G$ is the subgroup generated by all cyclic subnormal subgroups of $G$, and $G$ is a Baer group if it coincides with its Baer radical, or equivalently, if all finitely generated subgroups of $G$ are subnormal.

Lemma 2.8. Let $G$ be a group containing a torsion-free abelian normal subgroup which is not finitely generated, and let $B$ be the Baer radical of $G$. If every non-subnormal subgroup of $G$ is finitely generated, then the factor group $G / B$ is torsion-free.

Our next lemma is a relevant step in the proof of the main result of the paper.

Lemma 2.9. Let $G$ be a locally graded group with finitely many normalizers of non-polycyclic subgroups. Then either $G$ is polycyclic or its commutator subgroup $G^{\prime}$ is periodic.

Proof. The group $G$ is soluble-by-finite by Lemma 2.2. Assume that the statement is false, and choose a counterexample with a minimal number $k$ of proper normalizers of non-polycyclic subgroups. Then $k>0$ (see [8]), and of course it can be assumed without loss of generality that $G$ has no periodic non-trivial normal subgroup. Let $N_{G}\left(X_{1}\right), \ldots, N_{G}\left(X_{k}\right)$ be these normalizers. For every $i=1, \ldots, k$, the subgroup $N_{G}\left(X_{i}\right)$ has less than $k$ proper normalizers of non-polycyclic subgroups, and hence, $N_{G}\left(X_{i}\right)^{\prime}$ is periodic. On the other hand, the subgroup $N_{G}\left(X_{i}\right)$ has finitely many conjugates in $G$, so that the conjugacy class of $N_{G}\left(X_{i}\right)^{\prime}$ is also finite and hence, the normal closure $\left(N_{G}\left(X_{i}\right)^{\prime}\right)^{G}$ is periodic by Lemma 2.7. It follows that every $N_{G}\left(X_{i}\right)$ is a torsion-free abelian group. In particular, $N_{G}\left(X_{i}\right)$ is not contained in $N_{G}\left(X_{j}\right)$ for $i \neq j$. Thus, the subgroup $N_{G}\left(X_{i}\right)$ is either normal or self-normalizing in $G$, 
and in the latter case the index $\left|G: N_{G}\left(X_{i}\right)\right|$ is finite. Let $A_{i}$ be the core of $N_{G}\left(X_{i}\right)$ in $G$. Then either $A_{i}=N_{G}\left(X_{i}\right)$ or the index $\left|G: A_{i}\right|$ is finite, and in both cases the torsion-free abelian subgroup $A_{i}$ cannot be finitely generated. Moreover, as $X_{i}$ is not normal in $G$ and $G^{\prime}$ is infinite, we have that $A_{i}$ is not contained in $Z(G)$.

Let $x_{i}$ be an element of $G$ such that $\left[A_{i}, x_{i}\right] \neq\{1\}$, and put $B_{i}=\left\langle x_{i}, A_{i}\right\rangle$. Assume that the group $B_{i} /\left(\left\langle x_{i}\right\rangle \cap A_{i}\right)$ has periodic commutator subgroup, so that in particular, $\left\langle x_{i}\right\rangle \cap A_{i}=\left\langle x_{i}^{m_{i}}\right\rangle \neq\{1\}$. Clearly, $\left\langle x_{i}, B_{i}^{\prime}\right\rangle / Z\left(\left\langle x_{i}, B_{i}^{\prime}\right\rangle\right)$ is periodic, so that $\left\langle x_{i}, B_{i}^{\prime}\right\rangle^{\prime}$ is likewise periodic and hence, $\left[B_{i}^{\prime}, x_{i}\right]=\{1\}$, as $B_{i}^{\prime} \leq A_{i}$ is torsionfree. Thus, $\left[A_{i}, x_{i}\right]^{m_{i}}=\left[A_{i}, x_{i}^{m_{i}}\right]=\{1\}$ and so $\left[A_{i}, x_{i}\right]=\{1\}$. This contradiction shows that $B_{i} /\left(\left\langle x_{i}\right\rangle \cap A_{i}\right)$ has non-periodic commutator subgroup, so that the group $\left\langle x_{i}, A_{i}\right\rangle / C_{\left\langle x_{i}\right\rangle}\left(A_{i}\right)$ is also a counterexample, and hence, even a minimal counterexample. Replacing $G$ by $\left\langle x_{i}, A_{i}\right\rangle / C_{\left\langle x_{i}\right\rangle}\left(A_{i}\right)$, it can be assumed without loss of generality that $G=\langle x\rangle \ltimes A$, where $A$ is the normalizer of a non-polycyclic subgroup $X$ and $C_{\langle x\rangle}(A)=\{1\}$. Suppose that $x$ has finite order, so that by a further reduction we may even assume that $x$ has prime order. Clearly, $G$ is not locally nilpotent and hence, by Lemma 2.8 it contains a non-subnormal subgroup $Y$ which is not finitely generated. Thus, $N_{G}(Y) \neq A$ and so $G=\left\langle A, N_{G}(Y)\right\rangle$. On the other hand, the subgroup $A \cap N_{G}(Y)$ is contained in $Z(G)$ and the index $\left|G: N_{G}(Y)\right|$ must be finite, a contradiction since $G^{\prime}$ is infinite. Therefore, $x$ has infinite order and $G$ is torsion-free.

The above argument also shows that in any minimal counterexample $G$ the proper normalizers of non-polycyclic subgroups have infinite index and so they must be normal. In particular, all non-subnormal subgroups of $G$ are polycyclic, and hence, Lemma 2.8 yields that either $G$ is a Baer group or the Baer radical of $G$ coincides with $A$. In the latter case all proper normalizers of non-polycyclic subgroups of $G$ are contained in $A$, so that $A$ is the unique such normalizer and hence, $G$ is nilpotent by Lemma 2.6. On the other hand, if $G$ is a Baer group, we have that all subgroups of $G$ are subnormal and so $G$ is also nilpotent in this case (see [18]). Among all counterexamples obtained in this way, we can of course choose one $G$ with smallest nilpotency class $c$. Then the torsion-free group $G / Z(G)$ cannot be a counterexample and hence, it is either polycyclic or abelian. In order to prove that $G / Z(G)$ must be polycyclic, we can of course suppose that $G / Z(G)$ is abelian, so that $G$ has class 2 and in particular, $G^{\prime}=\{[a, x] \mid a \in A\}$.

Assume that $G^{\prime}$ is not polycyclic, so that it contains a proper non-polycyclic subgroup $K$ (see [8, Corollary 2.6]). Put $L=\langle x, K\rangle$. Then $G^{\prime} \leq L^{G}$ and $G^{\prime} \cap L=$ $K<G^{\prime}$, so that $L$ is not normal in $G$ and hence, the normalizer $N_{G}(L)$ is abelian. It follows that $A \cap N_{G}(L)$ is contained in $Z(G)$. Consider an element $a$ of $A$ such that $1 \neq[a, x] \in K$. As $[a, L]=\langle[a, x]\rangle \leq K<L$, we have that $a$ belongs to $A \cap N_{G}(L) \leq Z(G)$, contradicting the choice of $a$. Therefore, $G^{\prime}$ is polycyclic. Since all subgroups $N_{G}\left(X_{1}\right), \ldots, N_{G}\left(X_{k}\right)$ have infinite index in $G$, it follows from Lemma 2.4 that $N_{G}\left(X_{1}\right) \cup \cdots \cup N_{G}\left(X_{k}\right)$ is a proper subset of $G$. Let $g$ be an element of the set

$$
G \backslash \bigcup_{i=1}^{k} N_{G}\left(X_{i}\right) .
$$

As $C_{G}(g)=C_{G}\left(\left\langle g, G^{\prime}\right\rangle\right)$, the factor group $G / C_{G}(g)$ is isomorphic to a group of 
automorphisms of the polycyclic group $\left\langle g, G^{\prime}\right\rangle$, and hence, $G / C_{G}(g)$ is likewise polycyclic (see [14, Part 1, Theorem 3.27]). In particular, there exists a finitely generated subgroup $E$ of $G$ such that $G=C_{G}(g) E$ and $G^{\prime} \leq E$. Moreover, the factor group $G / C_{G}(E)$ is also polycyclic. As $C_{G}(g)$ is a non-polycyclic group whose non-normal subgroups are polycyclic, $C_{G}(g)$ is abelian. Thus, $C_{G}(g) \cap C_{G}(E)$ is contained in $Z(G)$, and so $G / Z(G)$ is polycyclic.

Let $H$ be any subgroup of $G$ which is not finitely generated. Then $H \cap Z(G)$ is not finitely generated, so that the factor group $G /(H \cap Z(G))$ has finitely many normalizers and hence, it is central-by-finite (see [13]). It follows that every subgroup of $G$ which is not finitely generated has finitely many conjugates (see [7, Proposition 3.4]), a contradiction since the index $\left|G: N_{G}\left(X_{i}\right)\right|$ is infinite for $i=1, \ldots, k$. This last contradiction completes the proof of the lemma.

Corollary 2.10. Let $G$ be a torsion-free locally graded group with finitely many normalizers of non-polycyclic subgroups. Then $G$ is either polycyclic or abelian.

Now we deal with the groups whose commutator subgroup has finite exponent.

Lemma 2.11. Let $G$ be a nilpotent group with finitely many normalizers of nonpolycyclic subgroups. If the commutator subgroup $G^{\prime}$ of $G$ has finite exponent, then $G / Z(G)$ is finite.

Proof. Assume for a contradiction that $G^{\prime}$ is infinite, and let $m$ be the smallest positive integer such that $G^{\prime} \cap Z_{m}(G)$ is infinite. Then $K=G^{\prime} \cap Z_{m-1}(G)$ is a finite normal subgroup of $G$ and $\left(G^{\prime} \cap Z_{m}(G)\right) / K=U_{1} / K \times U_{2} / K$, where both factors $U_{1} / K$ and $U_{2} / K$ are infinite. On the other hand, $U_{1}$ and $U_{2}$ are normal subgroups of $G$, and so the indices $\left|G^{\prime}: U_{1}\right|$ and $\left|G^{\prime}: U_{2}\right|$ are finite by Lemma 2.3 . This contradiction shows that $G^{\prime}$ is finite, and hence, $G / Z(G)$ is likewise finite by Lemma 2.5.

Lemma 2.12. Let $G$ be an abelian-by-(locally finite) group with finitely many normalizers of non-polycyclic subgroups. If the commutator subgroup $G^{\prime}$ of $G$ has finite exponent, then $G^{\prime} /\left(G^{\prime} \cap Z(G)\right)$ is finite.

Proof. Let $e$ be the exponent of $G^{\prime}$, and let $A$ be an abelian normal subgroup of $G$ such that $G / A$ is locally finite. Then $\{1\}=[A, G]^{e}=\left[A^{e}, G\right]$, so that $A^{e}$ is contained in $Z(G)$ and hence, the group $G / Z(G)$ is also locally finite. Clearly, $G / Z(G)$ has finitely many normalizers of infinite subgroups and so $G / Z(G)$ is either central-by-finite or a Černikov group (see [3]). It follows that $G / Z(G)$ has a finite commutator subgroup, and hence, $G^{\prime} /\left(G^{\prime} \cap Z(G)\right)$ is finite.

It is now possible to prove the main result of the paper.

Theorem 2.13. Let $G$ be a locally graded non-polycyclic group with finitely many normalizers of non-polycyclic subgroups. Then the commutator subgroup $G^{\prime}$ of $G$ is a Cernikov group and its divisible part is a primary group.

Proof. The group $G$ is soluble-by-finite by Lemma 2.2 and its commutator subgroup $G^{\prime}$ is periodic by Lemma 2.9. Thus, the set $T$ of all elements of finite order of $G$ 
is a subgroup and the factor group $G / T$ is abelian. Since $T$ has finitely many normalizers of infinite subgroups, it is central-by-finite (see [3]).

Assume for a contradiction that $G^{\prime}$ is not a Černikov group, and choose a counterexample such that the set $\left\{N_{G}\left(X_{1}\right), \ldots, N_{G}\left(X_{k}\right)\right\}$ of all proper normalizers of non-polycyclic subgroups has smallest order $k$, and of course, $k>0$ (see [8]). It follows from Lemma 2.3 that $T$ cannot contain two infinite $G$-invariant subgroups with finite intersection. In particular, $C=Z(T)$ has finitely many non-trivial primary components, and so there exists a prime number $p$ such that the $p$-component $C_{p}$ of $C$ has infinite socle $S_{p}$. Then again Lemma 2.3 yields that $G^{\prime}$ is a finite extension of an abelian group of exponent $p$, and hence, $G^{\prime}$ has finite exponent and $G^{\prime} \cap Z(G)$ is finite. It follows that $G^{\prime} /\left(G^{\prime} \cap Z(G)\right)$ is infinite and so by Lemma 2.12 the group $G$ cannot be abelian-by-finite. For each $i=1, \ldots, k$, the normalizer $N_{G}\left(X_{i}\right)$ has less than $k$ proper normalizers of non-polycyclic subgroups, so that $N_{G}\left(X_{i}\right)^{\prime}$ is a Černikov group and hence, it is even finite. Thus, the normal subgroup $\left\langle N_{G}\left(X_{1}\right)^{\prime}, \ldots, N_{G}\left(X_{k}\right)^{\prime}\right\rangle^{G}$ is likewise finite by Dietzmann's Lemma, and replacing $G$ by the factor group $G / N$ it can be assumed without loss of generality that $N_{G}\left(X_{i}\right)$ is abelian for every $i=1, \ldots, k$. As $G$ is not abelian-by-finite, the index $\left|G: N_{G}\left(X_{i}\right)\right|$ is infinite for each $i=1, \ldots, k$. On the other hand, $N_{G}\left(X_{i}\right)$ is a non-polycyclic subgroup of $G$ and $N_{G}\left(N_{G}\left(X_{i}\right)\right)$ has finite index in $G$, so that all subgroups $N_{G}\left(X_{1}\right), \ldots, N_{G}\left(X_{k}\right)$ are normal in $G$. In particular, every non-polycyclic subgroup of $G$ is subnormal with defect at most 2 .

Since $G^{\prime}$ is abelian-by-finite, another application of Lemma 2.12 gives that $G^{\prime \prime} /\left(G^{\prime \prime} \cap Z\left(G^{\prime}\right)\right)$ is finite, so that $G^{\prime} / Z\left(G^{\prime}\right)$ has a finite commutator subgroup and hence, $G^{\prime} / Z_{2}\left(G^{\prime}\right)$ is finite by Lemma 2.5 . Thus, $Z_{2}\left(G^{\prime}\right)$ is an infinite normal subgroup of $G$ and all subgroups of $G / Z_{2}\left(G^{\prime}\right)$ are subnormal with defect at most 2. It follows that $G / Z_{2}\left(G^{\prime}\right)$ is a nilpotent group, so that $G^{\prime}$ is nilpotent and $G$ is soluble. As the nilpotent subgroup $C_{G}\left(G^{\prime}\right)$ is central-by-finite by Lemma 2.11 , we obtain from Lemma 2.12 that $G / C_{G}\left(G^{\prime}\right)$ is not periodic, so that there exists an element $x$ of $G$ of infinite order such that $\langle x\rangle \cap C_{G}\left(G^{\prime}\right)=\{1\}$. In particular, the index $\left|\left\langle x, G^{\prime}\right\rangle: Z\left(\left\langle x, G^{\prime}\right\rangle\right)\right|$ is infinite and hence, Lemma 2.11 yields that $\left\langle x, G^{\prime}\right\rangle$ is not nilpotent. Thus, the subgroup $\langle x\rangle$ is not subnormal in $G$, so the Baer radical of $G$ is a proper subgroup. As $G$ does not contain infinite Černikov normal subgroups by Lemma 2.3 , it follows that $G$ is finitely generated (see [9, Theorem 3]) and so even polycyclic by Lemma 2.2. This contradiction proves that $G^{\prime}$ is a Cernikov group.

Suppose finally that $G^{\prime}$ is infinite, so that its divisible part $J$ has an infinite primary component $P$. As the factor group $G / P$ has finitely many normalizers, it follows that $G^{\prime} / P$ is finite. The theorem is proved.

It turns out from the classification of locally graded groups with polycyclic non-normal subgroups that if $G$ is a non-polycyclic group of this type, then the commutator subgroup $G^{\prime}$ is either finite or a finite extension of a group of type $p^{\infty}$ (for some prime number $p$ ). This property is no longer true in the case of groups with finitely many normalizers of non-polycyclic subgroups. In fact, let $A=\left\langle a_{n}\right| n \in$ $\left.\mathbb{N}_{0}, a_{0}=1, a_{n+1}^{3}=a_{n} \forall n \in \mathbb{N}_{0}\right\rangle$ and $B=\left\langle b_{n} \mid n \in \mathbb{N}_{0}, b_{0}=1, b_{n+1}^{3}=b_{n} \forall n \in \mathbb{N}_{0}\right\rangle$ be two groups of type $3^{\infty}$, and put $W=A \times B$. Now consider the automorphism $x$ 
of $W$ defined by $a_{n}^{x}=b_{n}$ and $b_{n}^{x}=a_{n}^{-1} b_{n}^{-1}$ for all $n \in \mathbb{N}_{0}$. The semidirect product $G=\langle x\rangle \ltimes W$ is a hypercentral 3-group with $G^{\prime}=W$, and $W$ is the unique proper normalizer of a non-polycyclic subgroup of $G$.

Corollary 2.14. Let $G$ be a residually finite-by-abelian group with finitely many normalizers of non-polycyclic subgroups. Then either $G$ is polycyclic or $G / Z(G)$ is finite.

Proof. Since $G$ is residually finite-by-abelian, its commutator subgroup $G^{\prime}$ is residually finite and hence, it follows from Theorem 2.13 that either $G$ is polycyclic or $G^{\prime}$ is finite. Moreover, in the latter case $G / Z(G)$ is finite by Lemma 2.5 .

\section{References}

[1] C. Casolo, Torsion-free groups in which every subgroup is subnormal, Rend. Circ. Mat. Palermo 50 (2001) 321-324.

[2] M. De Falco, F. de Giovanni, C. Musella, Groups with few non-normal subgroups, in: Ischia Group Theory (2006), World Sci. Publ., Hackensack, NJ, 2007, pp. 33-45.

[3] F. De Mari, F. de Giovanni, Groups with finitely many normalizers of non-abelian subgroups, Ricerche Mat. 55 (2006) 311-317.

[4] F. De Mari, F. de Giovanni, Groups with finitely many normalizers of subnormal subgroups, J. Algebra 304 (2006) 382-396.

[5] F. De Mari, F. de Giovanni, Groups with finitely many normalizers of infinite index, J. Algebra Number Theory Appl. 7 (2007) 83-95.

[6] F. De Mari, F. de Giovanni, Groups with finitely many normalizers of non-nilpotent subgroups, Math. Proc. Roy. Irish Acad. 107 (2007) 143-152.

[7] S. Franciosi, F. de Giovanni, L.A. Kurdachenko, On groups with many almost normal subgroups, Ann. Mat. Pura Appl. 169 (1995) 35-65.

[8] S. Franciosi, F. de Giovanni, M.L. Newell, Groups with polycyclic non-normal subgroups, Algebra Colloq. 7 (2000) 33-42.

[9] H. Heineken, L.A. Kurdachenko, Groups with subnormality for all subgroups that are not finitely generated, Ann. Mat. Pura Appl. 169 (1995) 203-232.

[10] S.K. Mahdavianary, A special class of three-Engel groups, Arch. Math. (Basel) 40 (1983) 193-199.

[11] B.H. Neumann, Groups covered by permutable subsets, J. London Math. Soc. 29 (1954) 236-248.

[12] B.H. Neumann, Groups with finite classes of conjugate subgroups, Math. Z. 63 (1955) 76-96.

[13] Y.D. Polovickiř, Groups with finite classes of conjugate infinite abelian subgroups, Soviet Math. (Izv. VUZ) 24 (1980) 52-59.

[14] D.J.S. Robinson, Finiteness Conditions and Generalized Soluble Groups, Springer, Berlin, 1972.

[15] G.M. Romalis, N.F. Sesekin, Metahamiltonian groups, Ural. Gos. Univ. Mat. Zap. 5 (1966) 101-106.

[16] G.M. Romalis, N.F. Sesekin, Metahamiltonian groups II, Ural. Gos. Univ. Mat. Zap. 6 (1968) $52-58$.

[17] G.M. Romalis, N.F. Sesekin, Metahamiltonian groups III, Ural. Gos. Univ. Mat. Zap. 7 (1969/1970) 195-199.

[18] H. Smith, Torsion-free groups with all subgroups subnormal, Arch. Math. (Basel) 76 (2001) 1-6. 